PAIN

\title{
Could TRPV1-targeted analgesia of OA pain still be feasible?
}

Systemic targeting of transient receptor potential cation channel subfamily $\mathrm{V}$ member 1 (TRPV1) in osteoarthritis (OA) pain is known to have limited therapeutic efficacy and to cause hyperthermia. However, new data from Sara Kelly and colleagues at the Arthritis Research UK Pain Centre, University of Nottingham, UK, suggest that targeting TRPV1 activity locally within the joint could represent a more feasible approach to analgesia in OA.

Although TRPV1 expression on sensory nerves had been previously established, Kelly et al. demonstrated considerable immunoreactivity towards TRPV1 in synovial tissue from patients with $\mathrm{OA}$ or $\mathrm{RA}$, whereas limited or no reactivity was detected in post-mortem non-arthritic control tissues. Interestingly, TRPV1 was mainly associated with mononuclear cells, particularly $\mathrm{CD} 68^{+}$macrophages. "These data suggest that the increased TRPV1 abundance in OA synovium may be due to inflammatory cell hyperplasia rather than increased expression by resident synovial cells," says Kelly. "One implication of this
4 ...local antagonism of joint TRPV1 activity attenuated pre-existing pain behaviour responses... 77

finding is that the inclusion of patients without an inflammatory component to their OA in clinical trials of TRPV1 antagonists might have concealed potential benefits of this approach on some OA pain phenotypes, an important consideration for future studies."

In a rat monoiodoacetate (MIA) model, which shares common pathological features and associated pain responses with human OA, levels of a TRPV1 ligand (and possibly mRNA expression of Trpv1) were also found to be increased in the knee joints, but not in the central nervous system, compared with control animals. Furthermore, functional upregulation of TRPV1 activity in the joint, but not the spinal cord, was associated with sensitization of mechanically-evoked neuronal responses, despite markedly increased TRPV1 expression in the spinal cord in the MIA model. "These finding support the notion that the joint is a critical site at which TRPV1 is upregulated in OA," claims Kelly. Supporting this theory, local antagonism of joint TRPV1 activity attenuated pre-existing pain behaviour responses to a similar degree to systemic inhibition, but without inducing TRPV1-mediated hyperthermia.

The authors acknowledge that the mechanisms by which synovitis and synovial TRPV1 contribute to OA pain remain obscure. Nevertheless, "our preclinical experimental evidence supports future targeted site-directed investigations of the therapeutic potential of TRPV1 antagonists for OA pain associated with synovitis, suggesting that this site can be targeted without on-target hyperthermic side effects," concludes Kelly.

David Killock

Original article Kelly, S. et al. Increased function of pronociceptive TRPV1 at the level of the joint in a rat model of osteoarthritis pain. Ann. Rheum. Dis. doi:10.1136/ annrheumdis-2013-203413 